\title{
WECIA GRAPH: VISUALIZATION OF CLASSIFICATION PERFORMANCE DEPENDENCY ON GRAYSCALE CONVERSION SETTING
}

\author{
Pavel Škrabánek ${ }^{1}$, Sule Yildirim Yayilgan ${ }^{2}$ \\ ${ }^{1}$ Brno University of Technology \\ Institute of Automation and Computer Science \\ Technická 2896/2, Brno \\ Czech Republic \\ pavel.skrabanek@vut.cz \\ ${ }^{2}$ Norwegian University of Science and Technology \\ Department of Information Security and Communication Technology \\ Po. Box. 191, 2802 Gjøvik \\ Norway \\ sule.yildirim@ntnu.no
}

\begin{abstract}
Grayscale conversion is a popular operation performed within image pre-processing of many computer vision systems, including systems aimed at generic object categorization. The grayscale conversion is a lossy operation. As such, it can significantly influence performance of the systems. For generic object categorization tasks, a weighted means grayscale conversion proved to be appropriate. It allows full use of the grayscale conversion potential due to weighting coefficients introduced by this conversion method. To reach a desired performance of an object categorization system, the weighting coefficients must be optimally setup. We demonstrate that a search for an optimal setting of the system must be carried out in a cooperation with an expert. To simplify the expert involvement in the optimization process, we propose a WEighting Coefficients Impact Assessment (WECIA) graph. The WECIA graph displays dependence of classification performance on setting of the weighting coefficients for one particular setting of remaining adjustable parameters. We point out a fact that an expert analysis of the dependence using the WECIA graph allows identification of settings leading to undesirable performance of an assessed system.
\end{abstract}

Keywords: computer vision, generic object categorization, grayscale conversion, weighted means grayscale conversion, classification, performance evaluation, data visualization

\section{Introduction}

Generic object categorization is one of the fundamental tasks of computer vision. Design of object categorization systems is a complex procedure that involves at least selection of features, selection of a classifier and setting of their parameters. Some form of image pre-processing is usually incorporated in the systems, too. Several consecutive operations are typically performed within image pre-processing. The operations are carried out to enhance the images for the feature extraction [18].

One of the favourite operations performed within image pre-processing is grayscale conversion. The main reasons for using grayscale conversion are to give an effort to reduce time-complexity of the systems, and to reduce the amount of training data required to achieve a good performance [17]. Object categorization systems with embedded grayscale conversion can be found in medicine [2,21], autonomous vehicles [3,37], agriculture [5,33], biology [16], manufacturing [23], security [1,20] etc.

The grayscale conversion performs a reduction of three-dimensional colour data into a two-dimensional space [30]. Thus, there is an infinite number of ways to accomplish the conversion. A variety of different grayscale conversion methods were proposed for RGB colour images [7,11, 14, 15, 17, 24, 29]. Most of these methods were optimized to produce perceptually plausible grayscale results and they may not provide good outputs for object categorization tasks [30].

For object categorization tasks, weighted means-based grayscale conversion methods proved to be appropriate [17]. These methods are essentially a weighted sum of the red, green and blue colour channels (hence the name weighted means grayscale conversion). They differ in setting of weights. Setting of the weights influences outputs of these methods and can consequently influence classification performance of object categorization 
systems. It means that the weights must be appropriately setup to reach desired classification performances of the systems [12].

Herein, we demonstrate that the weighted means grayscale conversion can significantly influence the performance of object categorization systems; and therefore, a search for an optimal setting of the system must be carried out in cooperation with an expert. To simplify the expert involvement, we developed a graph which displays the dependency of the classification performance on the setting of the grayscale conversion weights. Thus, we call the graph as WEighting Coefficients Impact Assessment (WECIA) graph. We based the WECIA graph on an idea behind ternary diagrams [13].

\section{Material and Methods}

\subsection{Object Categorization Systems}

Categorization of objects in images by hardware and software means is one of the fundamental tasks of computer vision [10]. The basic setting of this task is as follows. There is one object presented in an object image $I$ at the input of an object categorization system. According to the object presented in the image, the system assigns the image to one class from a finite set of predefined classes. The system learns to recognize objects on a set of labelled images [19].

The object categorization process performed in learned systems usually consists of three successive steps [18] which can be depicted as a vison pipeline using three consecutive blocks (Fig. 1). Within the image preprocessing step (first block), the object image $I$ is processed by several consecutive operations to enhance the image for feature extraction. In the following step, a feature vector $\mathbf{x}$ is extracted from the enhanced image $I^{*}$ by a feature descriptor. Finally, a class label $y$ is assigned to the object image $I$ by a classifier using the feature vector $\mathbf{x}$. Thus, the final performance of the classifier is strongly influenced by its precursors in the vision pipeline, i.e. by the image pre-processing and the feature descriptor.

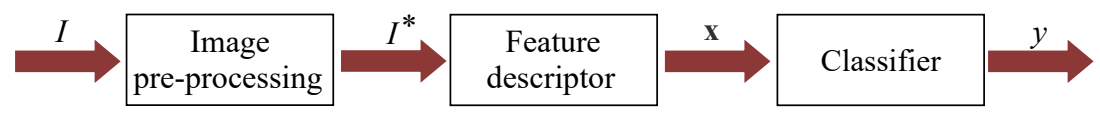

Figure 1: Basic vision pipeline of an object categorization system. The object image $I$ is enhanced within the image pre-processing. From the enhanced image $I^{*}$, a feature vector $\mathbf{x}$ is extracted by the feature descriptor. The classifier assigns a class label $y$ to the object image $I$ using the feature vector $\mathbf{x}$.

A final classification performance of an object categorization system is given by its structure (composition of the image pre-processing, selection of the feature descriptor and classifier, etc.) and by the setting of adjustable parameters. To ensure a desired classification performance of the system, an optimal setting of the parameters must be found. The parameter optimization can be fully automatic (e.g. using a robust parameter estimator [27], grid-search [6], random search [4]), or it can be carried out in cooperation with an expert observing the overall performance of the system [19].

The parameter optimization can be described as follows. An optimization algorithm generates various settings of system's adjustable parameters. For each setting, the object categorization system is trained and subsequently evaluated using performance measures. The evaluation can be carried out either using crossvalidation [9] or on an evaluation set [10]. The optimal setting is determined from the obtained set of evaluation results. In the case of an expert involvement into the search process, the optimal setting selection is influenced by the expert opinion and experience. The expert can use various visualization tools simplifying the expert assessment. In the case of the automatic search, the selection is entirely based on an objective function.

\subsection{Conversion of RGB Images to Grayscale Format}

Grayscale conversion transforms colour data into a single dimension. In standard photos and images, colour information is represented using RGB model [26]. An RGB object image $I$ is given as $I=\left(I_{R}, I_{G}, I_{B}\right)$, where $I_{R}, I_{G}$ and $I_{B}$ are its red, green and blue components, respectively. Let us further consider RGB object images as the input of the object categorization system (Fig. 1).

For the object categorization, the weighted means grayscale conversion is appropriate [17]. For the RGB object image $I=\left(I_{R}, I_{G}, I_{B}\right)$ of spatial resolution $M \times N$ pixels (px), this conversion method is given as

$$
Y=w_{R} I_{R}+w_{G} I_{G}+w_{B} I_{B}
$$

where $w_{R}, w_{G}$ and $w_{B}$ are weighting coefficients of the red, green and blue components, respectively [26]. The weighting coefficients $w$ determine proportions of the colour components in the resulting grayscale image $Y$. It holds that $w_{R}, w_{G}, w_{B} \in[0,1]$ and $w_{R}+w_{G}+w_{B}=1$. Resolution of the grayscale image $Y$ is $M \times N$ px. 


\subsection{Classification Performance Measures}

The basic requirement of performance of object categorization systems is the classification of unknown object images with minimum error [19]. A real performance of an object categorization system can be determined using performance measures [25]. Various single value performance measures $\mu$ can be used for this purpose. Each performance measure quantifies the performance from a different perspective.

To explain the idea behind the measures, let us consider the simplest case when only two classes, "positive" or simply $P$, and "negative" or simply $N$, are expected within the classification. For each object image, the decision-making process falls into one of four possible scenarios: the image is "positive" and the classifier correctly recognizes it as such ("true positive" or simply TP); the image is "negative" and the classifier correctly recognizes it as such ("true negative" or simply $T N$ ); the image is "positive" but the classifier labels it as "negative" ("false negative" or simply $F N$ ); or the image is "negative" but the classifier labels it as "positive" ("false positive" or simply FP) [31].

On the basis of the presented scenario, four fundamental quantities are formulized for the measurement of a binary classifier performance: number of "true positive" $|T P|$; number of "true negative" $|T N|$; number of "false negative" $|F N|$; and number of "false positive" $|F P|$ classifications. The quantities are usually summarized into a confusion matrix. The confusion matrix can be similarly constructed for multiclass problems [25].

A confusion matrix contains all necessary data for the classification performance evaluation, but working with it is complex (especially for multiclass problems). Thus, single value measures based on the confusion matrix are usually used instead of the confusion matrix itself [22]. From the best-known single value performance measures aimed at binary classifier evaluation, let us mention at least

$$
\begin{gathered}
\text { accuracy }=\frac{|T P|+|T N|}{|T P|+|F P|+|T N|+|F N|}, \\
\text { precision }=\frac{|T P|}{|T P|+|F P|}, \\
\text { recall }=\frac{|T P|}{|T P|+|F N|} .
\end{gathered}
$$

\subsection{Ternary Diagrams}

A ternary diagram is a graph which consists of an equilateral triangle in which a given plotted point represents the relative proportions $(a, b, c)$ of three end-members $(\mathrm{A}, \mathrm{B}$ and $\mathrm{C})$, usually expressed as percentages. Moreover, the sum of the relative proportions is equal to a given value, e.g. for percentages, it holds that $a+b+c=1$ $(a+b+c=100 \%)[13]$.

Let us consider a relationship $z=100 a b c$, where $a+b+c=1$ and $a, b, c \in[0,1]$. The ternary diagram allows to display the relationship in the full range of independent variable values. The dependent variable $z$ can be represented in different ways, e.g. using shaded surface (Fig. 2 (a)), as contour plot (Fig. 2 (b)), and as scatter plot (Fig. $2(\mathrm{c})$ ). In all cases, $z$ variable values are expressed using colour, and they can be estimated from a colour bar.

The meaning of the axes in a ternary diagram is as follows. The axis related to the member A is the left edge of the triangle. The relative proportion of $\mathrm{A}, a$, is plotted on the axis where $a$ increases downwards. The same principle is used for the remaining two axes, where the bottom edge is related to the component B and the right one to the component $\mathrm{C}$. The relative proportion of $\mathrm{B}$ increases in the right direction, and the relative proportion of $\mathrm{C}$ upwards.

Reading of a dependent variable value for a specific combination of independent variable values is carried out using auxiliary lines. In Fig. 3, the $z$ value for $a=0.1, b=0.2$ and $c=0.7$ is determined using the lines (dashed lines). Each of the lines is parallel with one of the triangle's edges. We named the lines with respect to referred components; e.g. the "A line" is related to the component A. The lines intersect the edges at the examined values of the independent variables, e.g. the "A line" intersects the left edge at 0.1. Intersection of the auxiliary lines determines the desired value of $z$. The $z$ value can be estimated using the colour bar. In this case, the value is around 1.4.

Construction of the diagram follows the process of reading, but naturally, executed in reverse order. The position of a point can be easily expressed explicitly. Taking the mid-point of the base $b$ as the origin of the Cartesian system (see Fig. 3), location of a point in the Cartesian system is given as $\left(b+\frac{1}{2} c-\frac{1}{2}, \frac{\sqrt{3}}{2} c\right)$. Information necessary for construction of the various types of the diagram can be found e.g. in [36]. 


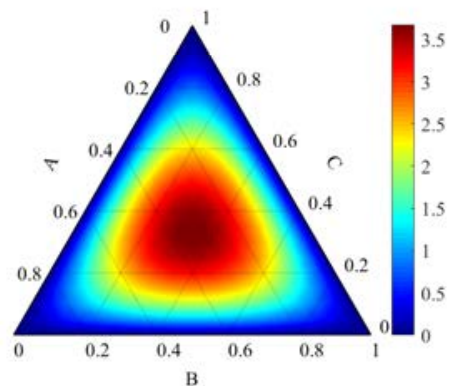

(a)

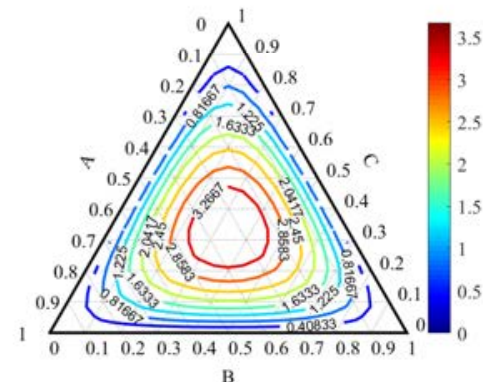

(b)

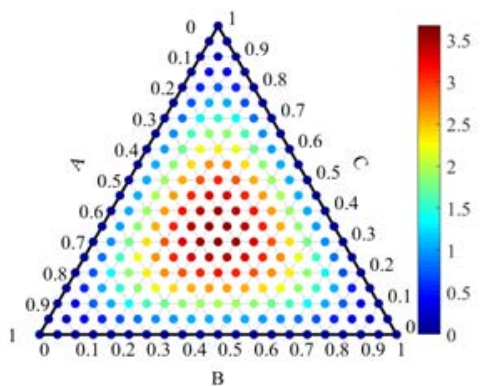

(c)

Figure 2: Ternary diagrams displaying a relationship $z=100 a b c$ for $a, b, c \in[0,1]$, where $a+b+c=1$. The independent variables $a, b$, and $c$ are relative proportions of three end-members $\mathrm{A}, \mathrm{B}$ and $\mathrm{C}$, respectively. The diagrams differ in representation of the dependent variable values. The relationship is displayed (a) using shaded surface, (b) as colour contour plot, and (c) as coloured scatter plot. In all three cases, the colour shades correlate with the dependent variable values, where the values can be estimated using a colour bar.

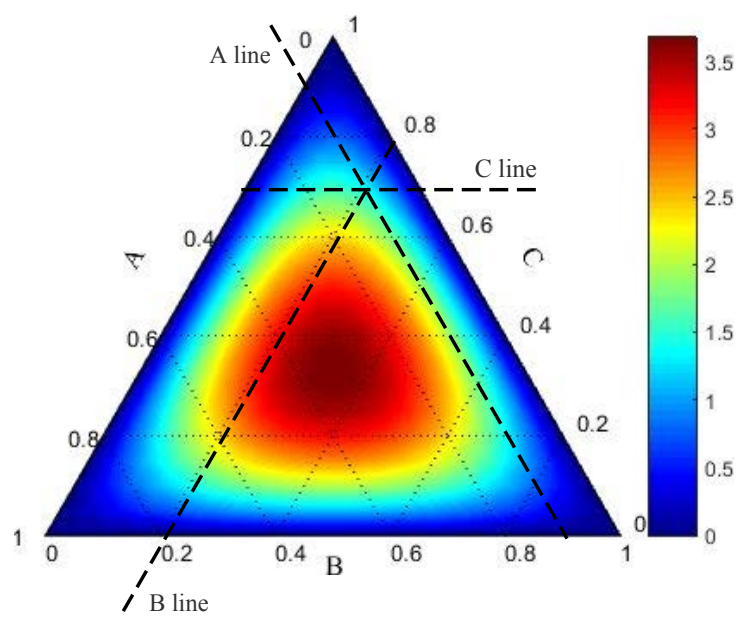

Figure 3: A dependent variable value can be read off using auxiliary lines (dashed lines). In this case, the $z$ value for $a=0.1, b=0.2$ and $c=0.7$ is determined. Intersection of the auxiliary lines determines a point in the triangle surface. Colour of the point corresponds to the dependent variable value. The value can be estimated using the colour bar, i.e. $z=1.4$ in this case.

\subsection{WECIA Graph}

Classification performance of an object categorization system is, among others, influenced by setting of its adjustable parameters; however, effect of individual parameters on the performance is uneven. When integrated in an object categorization system, the weighted means grayscale conversion (1) gives three adjustable parameters (weighting coefficients $w$ ) into the system. We hypothesize that the coefficients $w_{R}, w_{G}$, and $w_{B}$ can meaningfully influence classification performance of such a system.

To confirm the hypothesis, dependency of the classification performance on the weighting coefficients $w$ needs to be analysed by a human expert. The analysis must be carried out in the whole ranges of the coefficients. To simplify the analysis, we propose the WECIA graph. The graph is aimed to display dependency of a single value performance measure $\mu$ on the weighting coefficients $w_{R}, w_{G}$, and $w_{B}$ for $\forall w_{R}, w_{G}, w_{B} \in[0,1]$.

The WECIA graph is a ternary diagram, where the colour components $I_{R}, I_{G}$, and $I_{B}$ are the three endmembers of the diagram. The weighting coefficients $w_{R}, w_{G}$, and $w_{B}$ represent the relative proportions of $I_{R}, I_{G}$, and $I_{B}$, respectively. Performance measure values provided by the measure $\mu$ are displayed using colour in the area inside the triangle.

Construction of the WECIA graph for an object categorization system requires evaluation of the system for various settings of the weighting coefficients $w$. Let us denote a setting of the weighting coefficients as an ordered triple $\left(w_{R}, w_{G}, w_{B}\right)$. The evaluation of the system should be performed for all possible settings 
$\left(w_{R}, w_{G}, w_{B}\right)$ which is naturally not feasible in practice. Thus, when constructing the WECIA graph, we carry out the evaluation for a finite set of coefficient settings $D$, and we estimate the unknown evaluation results using an interpolation function to provide a graphic output suitable for human assessment.

To get an expressive image of the weighting coefficient influence on the performance, the evaluation results should uniformly cover the surface bounded by the ternary diagram. It means that each weighting coefficient $w$ in $\left(w_{R}, w_{G}, w_{B}\right)$ can take any value from $\{0, \Delta w, 2 \Delta w, \ldots, 1\}$, but combinations of the coefficients $w$ in $\left(w_{R}, w_{G}, w_{B}\right)$ are bounded by the condition $w_{R}+w_{G}+w_{B}=1$. All settings $\left(w_{R}, w_{G}, w_{B}\right)$ satisfying these conditions form the set $D$. In our approach, the step $\Delta w$ is determined by a desired number of points on one axis of the diagram $n$, where $\Delta w=(n-1)^{-1}$ and $n \in \mathbb{N}^{+}$.

To construct a WECIA graph, classification performance of the system must be evaluated using the measure $\mu$ for $\forall\left(w_{R}, w_{G}, w_{B}\right) \in D$. For each setting $\left(w_{R}, w_{G}, w_{B}\right) \in D$, the training-evaluation process must be carried out separately. Setting of the remaining adjustable parameters (introduced by the classifier, by the feature detector, and by other operations performed within the image pre-processing) must be identical for all these training-evaluation processes. Thus, the WECIA graph displays a dependency of the measure $\mu$ on the weighting coefficients $w$ for one specific setting of the remaining adjustable parameters.

We use a function tersurf from ternary plots package [28] for MATLAB to plot the WECIA graph. This function generates a shaded surface ternary diagram, i.e. it ensures the interpolation of unknown evaluation results. We use default setting of this function.

\subsection{Case Study}

We demonstrate the usage of the WECIA graphs on a grape detector. The grape detector is an object categorization system aimed at recognition of single grape images [32], i.e. grape detection is a binary classification task. The grape detector is based on a histogram of oriented gradients (HOG) descriptor [8] and a support vector machine (SVM) classifier with a radial basis function (RBF) kernel. Within the image pre-processing, only the weighted means grayscale conversion (1) is performed, i.e. $I^{*}=Y$.

The grape detector has several adjustable parameters. The parameters associated with the grayscale conversion are the weighting coefficients $w_{R}, w_{G}$, and $w_{B}$. The SVM classifier uses a regularization constant and the width of the RBF kernel is represented by $\sigma$. In the HOG descriptor, five additional parameters must be setup [34]. Within this case study, the HOG descriptor has the following setting: linear gradient voting into 9 bins in $0^{\circ}-180^{\circ} ; 6 \times 6$ px blocks; $2 \times 2$ px cells; 2 overlapping cells between adjacent blocks. The regularization constant of the classifier is 10 . We consider two randomly selected settings of the kernel width $\sigma$, i.e. WECIA graphs for two different settings of the detector must be constructed. Specifically, $\sigma \in\{1,10\}$ in this case study.

For the construction of the WECIA graphs, we train and evaluate the detector on sets T-01 [35] and EX01 [34], respectively. The desired number of points on one axis is $n=20$. We evaluate the detector using accuracy (2a), precision $(2 \mathrm{~b})$ and recall (2c), i.e. three WECIA graphs are generated for each $\sigma$ setting.

\section{Results}

WECIA graphs obtained for the grape detector with kernel of width $\sigma=1$ are shown in Fig. 4 . Even with a little change in weighting coefficient setting, accuracy (Fig. 4 (a)), precision (Fig. 4 (b)) and recall (Fig. 4 (c)) show sudden and steep changes in the performance measure values (sudden changes in colour). The changes were about $45 \%, 90 \%$ and $100 \%$ in accuracy, precision and recall, respectively. The highest registered accuracy, precision and recall was about $95 \%, 90 \%$ and $100 \%$, respectively.
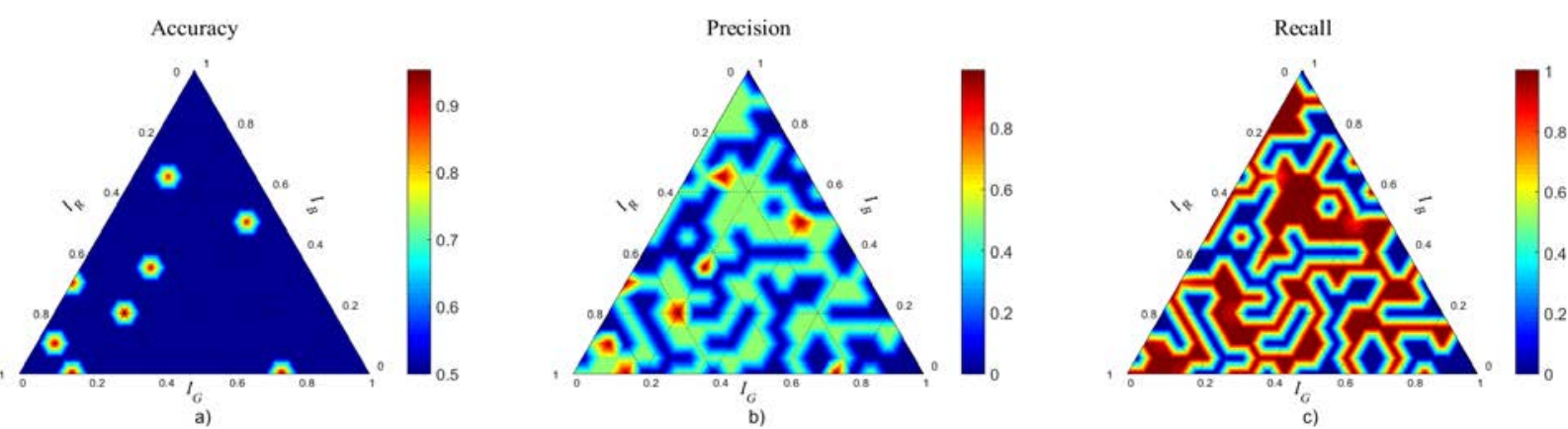

Figure 4: WECIA graphs showing (a) accuracy, (b) precision and (c) recall of the grape detector trained on the set T-01 and evaluated on the set EX-01 for $\sigma=1$. 
Graphs obtained for the grape detector with kernel of width $\sigma=10$ show gradual changes in accuracy (Fig. 5 (a)), precision (Fig. 5 (b)) and recall (Fig. 5 (c)). The highest registered accuracy, precision and recall was about $79 \%, 100 \%$ and $57 \%$, respectively. Total changes in accuracy, precision and recall was about $4 \%$, $0 \%$, and $8 \%$, respectively.
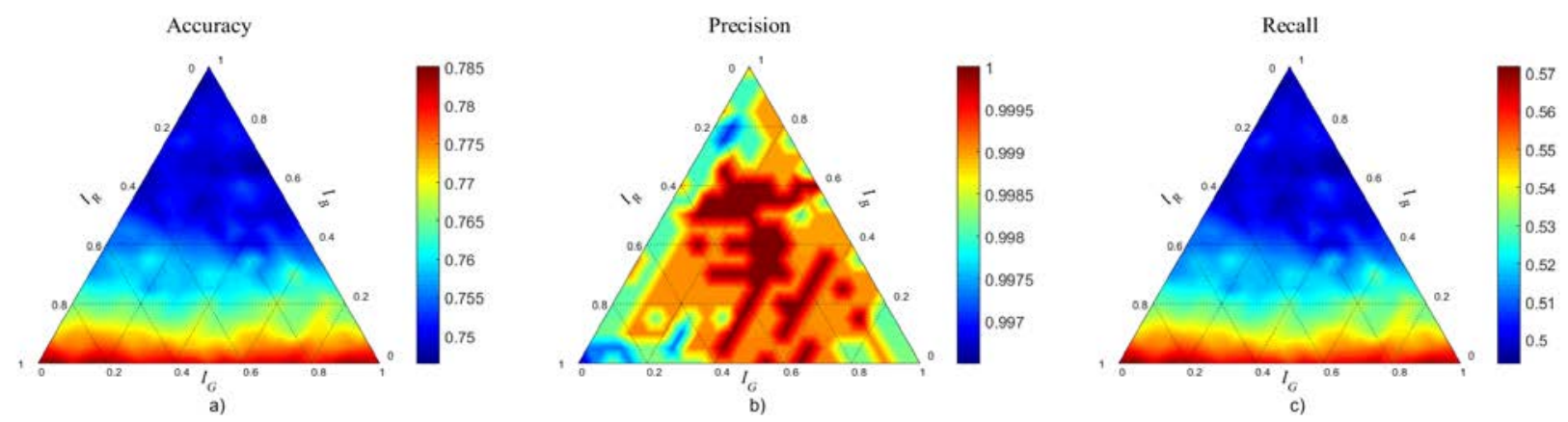

Figure 5: WECIA graphs showing (a) accuracy, (b) precision and (c) recall of the grape detector trained on the set T-01 and evaluated on the set EX-01 for $\sigma=10$.

\section{Discussion}

We hypothesized that the weighting coefficients $w$ can meaningfully influence classification performance of object categorization systems with embedded grayscale conversion (1). We examined the hypothesis on the case study. We constructed WECIA graphs displaying dependence of accuracy, precision and recall for two different settings of kernel width $\sigma$. The WECIA graphs obtained for $\sigma=1$ (Fig. 4) as well as the graphs obtained for $\sigma=10$ (Fig. 5) display correlations between the performance measures and the weighting coefficients $w$. With respect to this fact, we consider the hypothesis to be confirmed and we further interpret the results in the WECIA graphs.

We registered the highest accuracy, precision and recall for the grape detector with $\sigma=1$. The highest accuracy $94.80 \%$ was recorded for $w_{R}=0.05, w_{G}=0.95$ and $w_{B}=0.00$ (Fig. 4 (a)); however, accuracy of the detector was only $50 \%$ for all the neighbour settings of the weighting coefficients. Several other weighting coefficient settings providing accuracy above $90 \%$ can be found in the WECIA graph (Fig. 4 (a)), but the fall of accuracy on $50 \%$ for the neighbour settings can be observed for all of them. Moreover, accuracy of the grape detector is $50 \%$ for majority of the settings. We observed the same trend also for precision (Fig. 4 (b)) and recall (Fig. 4 (c)). Thus, we consider the high values of the performance measures to be anomalies, and we recommend avoiding all settings containing the combination of $\sigma=1$ with the settings of remaining adjustable parameters as specified in subsection 2.6.

For the kernel of width $\sigma=10$, we recorded the highest accuracy $78.13 \%$ for $w_{R}=0.95, w_{G}=0.05$ and $w_{B}=0.00$ (Fig. 5 (a)). For all neighbour settings of the weighting coefficients, accuracy did not fall under $77 \%$. We observed that the accuracy descends with increasing proportion of the blue component in the resulting grayscale image, and all changes in accuracy are consistent in the context of the displayed settings. The same conclusion can be made for recall (Fig. 5 (c)). As the total change in precision is only $0.35 \%$ (Fig. 5 (c)), we considered this performance measure to be resistant to changes in the weighting coefficient settings. With respect to all these facts, the grape detector with $\sigma=10$ seems to be robust to small changes in colour space. Thus, settings containing the combination of $\sigma=10$ with the settings of remaining adjustable parameters (see subsection 2.6) should be taken into account when searching for the optimal setting of the detector.

\section{Conclusion}

We demonstrated that the weighted means grayscale conversion has the capability to influence classification performance of object categorization systems. The performance is dependent on setting of the weighting coefficients. We established that the dependence must be analysed by an expert to avoid undesirable performance. We designed the WECIA graph as a tool simplifying the expert analysis. We showed that robustness of object categorization systems to changes in the colour space can be estimated using WECIA graphs. Robust settings feature with gradual changes in performance with gradual change of weighting coefficient setting. An unstable setting is characteristic with rapid substantial changes in performance even for small changes in weighting coefficient setting (e.g. $45 \%$ variance in accuracy for $\Delta w=0.05$ ). We expect that WECIA graphs have a much 
wider application potential in computer vision, e.g. when analysing influence of the weighting coefficient setting on image segmentation in systems with embedded weighted means grayscale conversion.

Acknowledgement: This work was supported by The Ministry of Education, Youth and Sports of the Czech Republic, INTER-COST project LTC18053 and European COST Action CA15140.

\section{References}

[1] Aubin, V., Mora, M.: A new descriptor for person identity verification based on handwritten strokes off-line analysis. Expert Systems with Applications 89, 241 - 253 (2017). DOI https://doi.org/10.1016/j.eswa.2017.07.039

[2] Bankhead, P., Scholfield, C.N., McGeown, J.G., Curtis, T.M.: Fast retinal vessel detection and measurement using wavelets and edge location refinement. PLOS ONE 7(3), 1-12 (2012). DOI 10.1371/journal.pone.0032435

[3] Barbu, T.: Pedestrian detection and tracking using temporal differencing and HOG features. Computers \& Electrical Engineering 40(4), 1072 - 1079 (2014). DOI http://dx.doi.org/10.1016/j.compeleceng.2013.12.004

[4] Bergstra, J., Bengio, Y.: Random search for hyper-parameter optimization. The Journal of Machine Learning Research 13, 281 - 305 (2012)

[5] Burgos-Artizzu, X.P., Ribeiro, A., Tellaeche, A., Pajares, G., Fernández-Quintanilla, C.: Analysis of natural images processing for the extraction of agricultural elements. Image and Vision Computing 28(1), 138 149 (2010). DOI http://dx.doi.org/10.1016/j.imavis.2009.05.009

[6] Coope, I.D., Price, C.J.: On the convergence of grid-based methods for unconstrained optimization. SIAM Journal on Optimization 11(4), 859-869 (2001)

[7] Cui, M., Hu, J., Razdan, A., Wonka, P.: Color-to-gray conversion using ISOMAP. The Visual Computer 26(11), 1349-1360 (2010). DOI 10.1007/s00371-009-0412-7

[8] Dalal, N., Triggs, B.: Histograms of oriented gradients for human detection. In: Computer Vision and Pattern Recognition, 2005. CVPR 2005. IEEE Computer Society Conference on, vol. 1, pp. 886-893 (2005)

[9] Forman, G., Scholz, M.: Apples-to-apples in cross-validation studies: Pitfalls in classifier performance measurement. SIGKDD Explor. Newsl. 12(1), 49-57 (2010). DOI 10.1145/1882471.1882479

[10] Gauman, K., Leibe, B.: Visual Object Recognition. Synthesis Lectures on Artificial Intelligence and Machine Learning. Morgan \& Claypool Publishers (2010)

[11] Grundland, M., Dodgson, N.A.: Decolorize: Fast, contrast enhancing, color to grayscale conversion. Pattern Recognition 40(11), 2891-2896 (2007)

[12] Güneş, A., Kalkan, H., Durmuş, E.: Optimizing the color-to-grayscale conversion for image classification. Signal, Image and Video Processing 10(5), 853-860 (2016). DOI 10.1007/s11760-015-0828-7

[13] Howarth, R.J.: Sources for a history of the ternary diagram. The British Journal for the History of Science 29, 337-356 (1996)

[14] Hsin, C., Le, H.N., Shin, S.J.: Color to grayscale transform preserving natural order of hues. In: Proceedings of the 2011 International Conference on Electrical Engineering and Informatics, pp. 1-6 (2011). DOI 10.1109/ICEEI.2011.6021794

[15] ITU-R Recommendation BT.601: Studio encoding parameters of digital television for standard 4:3 and wide screen $16: 9$ aspect ratios (2011)

[16] Jin, T., Hou, X., Li, P., Zhou, F.: A novel method of automatic plant species identification using sparse representation of leaf tooth features. PLOS ONE 10(10), 1-20 (2015). DOI 10.1371/journal.pone.0139482

[17] Kanan, C., Cottrell, G.W.: Color-to-Grayscale: Does the Method Matter in Image Recognition? PLoS ONE 7(1), 1-7 (2012)

[18] Krig, S.: Computer Vision Metrics: Survey, Taxonomy, and Analysis, 1st edn. Apress, Berkely, CA, USA (2014)

[19] Leondes, C.: Image Processing and Pattern Recognition, vol. 5. Academic Press (1998)

[20] Lionnie, R., Alaydrus, M.: A comparison of human skin color detection for biometrie identification. In: 2017 International Conference on Broadband Communication, Wireless Sensors and Powering (BCWSP), pp. 1-5 (2017). DOI 10.1109/BCWSP.2017.8272565

[21] Pennisi, A., Bloisi, D.D., Nardi, D., Giampetruzzi, A.R., Mondino, C., Facchiano, A.: Skin lesion image segmentation using delaunay triangulation for melanoma detection. Computerized Medical Imaging and Graphics 52, 89 - 103 (2016). DOI http://dx.doi.org/10.1016/j.compmedimag.2016.05.002

[22] Powers, D.M.W.: Evaluation: From precision, recall and f-measure to ROC, informedness, markedness \& correlation. Journal of Machine Learning Technologies 2(1), 37-63 (2011) 
[23] Raghuwanshi, G., Tyagi, V.: Texture image retrieval using adaptive tetrolet transforms. Digital Signal Processing 48, 50 - 57 (2016). DOI http://dx.doi.org/10.1016/j.dsp.2015.09.003

[24] Rasche, K., Geist, R., Westall, J.: Re-coloring images for gamuts of lower dimension. Computer Graphics Forum 24(3), 423-432 (2005)

[25] Sokolova, M., Lapalme, G.: A systematic analysis of performance measures for classification tasks. Information Processing \& Management 45(4), 427 - 437 (2009). DOI https://doi.org/10.1016/j.ipm.2009.03.002

[26] Solomon, C., Breckon, T.: Fundamentals of Digital Image Processing: A Practical Approach with Examples in Matlab, 1st edn. Wiley Publishing (2011)

[27] Stewart, C.V.: Robust parameter estimation in computer vision. SIAM Review 41(3), $513-537$ (1999)

[28] Theune, U.: Ternary plots. https://www.mathworks.com/matlabcentral/fileexchange/7210-ternary-plots (2002-2005)

[29] Tirui Wu, A.T.: Color-to-grayscale conversion through weighted multiresolution channel fusion. Journal of Electronic Imaging 23, 23 - 23 - 6 (2014). DOI 10.1117/1.JEI.23.4.043004

[30] Čadík, M.: Perceptual evaluation of color-to-grayscale image conversions. Computer Graphics Forum 27(7), 1745-1754 (2008). DOI 10.1111/j.1467-8659.2008.01319.x

[31] Škrabánek, P., Doležel, P.: On reporting performance of binary classifiers. Scientific Papers of the University of Pardubice, Series D XXIV, 181-192 (2017)

[32] Škrabánek, P., Doležel, P.: Robust grape detector based on SVMs and HOG features. Computational Intelligence and Neuroscience 2017, 1-17 (2017). DOI 10.1155/2017/3478602

[33] Škrabánek, P., Majerík, F.: Simplified version of white wine grape berries detector based on SVM and HOG features. In: Proceedings of the 5th Computer Science On-line Conference 2016 (CSOC2016). Springer International Publishing (2016). In press

[34] Škrabánek, P., Majerík, F.: Detection of grapes in natural environment using HOG features in low resolution images. Journal of Physics: Conference Series 870(1), 012,004 (2017)

[35] Škrabánek, P., Runarsson, T.P.: Detection of grapes in natural environment using support vector machine classifier. In: Proceedings of the 21st International Conference on Soft Computing MENDEL 2015, pp. 143-150. Brno University of Technology, Brno, Czech Republic (2015)

[36] Wright, H.: Introduction to scientific visualization. Springer (2007)

[37] Yoo, H., Yang, U., Sohn, K.: Gradient-enhancing conversion for illumination-robust lane detection. IEEE Transactions on Intelligent Transportation Systems 14(3), 1083-1094 (2013) 